\title{
Icariin stimulates differentiation of bone marrow-derived mesenchymal stem cells (BM-MSCs) through activation of $\mathrm{CAMP} / \mathrm{PKA} / \mathrm{CREB}$
}

\author{
Dan Lou ${ }^{1}$, Jifeng Ye ${ }^{1}$, Lianhua Yang${ }^{1}$, Zheng Wu ${ }^{\circledR 2}$, Wei Zheng ${ }^{2}$, Hui Zhang ${ }^{*}$ \\ ${ }^{I}$ The Second Affiliated Hospital and Yuying Children's Hospital of Wenzhou Medical University, Wenzhou, China, \\ ${ }^{2}$ Harbin University of Commerce, Harbin, China
}

\begin{abstract}
Icariin, a prenylated flavonol glycoside isolated from Epimedium, has been considered as a potential alternative therapy for osteoporosis. The present study aimed to clarify the detailed molecular mechanisms of action of icariin on osteoblast function, using bone marrow-derived mesenchymal stem cells (BM-MSCs). BM-MSCs were first stimulated by icariin. Then, gene and protein expression of cAMP/ PKA/CREB signaling molecules were analyzed by RT-PCR and western blotting (WB), and alkaline phosphatase (ALP) was analyzed in cell lysates by ELISA. MTT assays indicated that icariin did not have significant effects on cell viability up to $1 \mu \mathrm{M}$. Icariin showed a dose-dependent effect on the alkaline phosphatase activity of BM-MSCs. WB analysis showed that icariin treatment of BM-MSCs significantly enhanced the protein expression of protein kinase A (PKA) and cAMP-responsive element binding protein (CREB), while RT-PCR results showed that icariin dose-dependently increased the mRNA levels of $P K A$ and $C R E B$. Icariin induced BM-MSC differentiation by BMP2, Smad1, and Runx2. RT-PCR and WB results indicated that icariin significantly increased the expression of BMP2, Smad1, and Runx2 in BM-MSCs. These results suggest that icariin is an agonist of the cAMP/PKA/CREB pathway in BM-MSC differentiation, raising the possibility that it could be used in the treatment of osteoporosis.
\end{abstract}

Keywords: Icariin/molecular mechanisms. BM-MSCs. cAMP. PKA. CREB.

\section{INTRODUCTION}

Osteoporosis is characterized by low bone mineral density (BMD) and loss of the structural and biomechanical properties that are required to maintain bone homeostasis (Ivanova et al., 2015). Icariin is the main active flavonoid glucoside of Epimedium pubescens, which has been reported to enhance bone healing and reduce the incidence of osteoporosis (Hsieh et al., 2010). Icariin restores the osteogenic differentiation and bone formation of bone marrow stromal cells in a rat model of estrogen deficiency-induced osteoporosis (Luo et al., 2015). In the ovariectomized (OVX) calvarial defect model, icariin loaded on CPC scaffolds enhanced both osteogenesis and angiogenesis, while a system of local sustained release of icariin combined with systemic administration achieved a better effect on bone defect regeneration (Wu et al., 2017).

\footnotetext{
*Correspondence: H. Zhang. The Second Affiliated Hospital and Yuying Children's Hospital, Wenzhou Medical University, Wenzhou, China. E-mail: zhanghui86577@163.com
}

Osteoblasts, osteocytes and osteoclasts are the three types of bone cells that are in direct contact with all the cellular elements in the bone marrow (Carrington, 2005). Mesenchymal stem cells (MSCs) are a promising source of cells for use in cell-based therapeutics and regenerative medicine due to their ability to self-renew and differentiate into a number of functional cell types. To date, bone marrow-derived mesenchymal stem cells (BM-MSCs) have been the most widely-studied family of stem cells (Li et al., 2016). BM-MSCs have been estimated to represent $0.01-0.0001 \%$ of the nucleated cells in adult human bone marrow (Dazzi et al., 2006). BM-MSCs are progenitors of skeletal tissue components such as bone, cartilage, the hematopoiesis-supporting stroma, and adipocytes (Bianco, Riminucci, Gronthos, 2001). Bone morphogenesis protein-2 (BMP-2), a member of the BMP family, is one of the best-characterized inducers of osteochondrogenesis and can increase the expression of markers of both osteo- and chondrogenic lineage differentiation of progenitor cells, such as MSCs. It has recently been shown that protein kinase $\mathrm{A}(\mathrm{PKA})$ activation 
also forms an important component of the mechanism by which BMP-2 mediates MSC differentiation. Once activated, PKA usually phosphorylates its prototypical downstream transcription factor, cAMP-responsive element binding protein (CREB), which is associated with a number of important physiological functions including osteochondrogenic differentiation (Zhao, Li, Zhou, 2009).

Icariin has been shown to have an anti-osteoporotic effect by regulating the bidirectional differentiation of BM-MSCs through the canonical Wnt signaling pathway (Huang et al., 2017). Icariin is also a bone anabolic agent that may exert its osteogenic effects through the induction of BMP-2 and NO synthesis, subsequently regulating expression of $\mathrm{Cbfa} 1 / \mathrm{Runx} 2, \mathrm{OPG}$, and RANKL. This effect may contribute to its effect on the induction of osteoblast proliferation and differentiation, resulting in bone formation (Hsieh et al., 2010). In this study, we clarified the detailed molecular mechanisms of action of icariin on the osteoblastic function of BMMSCs. The results demonstrate that icariin induces osteoblast differentiation via BMP2, through activation of cAMP/PKA/CREB signaling in osteoblasts.

\section{MATERIAL AND METHODS}

\section{Material and reagents}

Icariin was obtained from Sigma-Aldrich (St. Louis, MO, USA) and the purity of the compound was more than $99 \%$. Stock solutions of icariin were prepared in dimethyl sulfoxide (DMSO) (Sigma-Aldrich). In all studies, the final concentration of DMSO was $0.1 \%(\mathrm{v} / \mathrm{v})$. The icariin stock solutions were stored at $-20^{\circ} \mathrm{C}$.

\section{Cell culture}

BM-MSCs were purchased from Saiye Biological Technology Co., Ltd. (Guangzhou, China). BM-MSCs were cultured in $25 \mathrm{~cm}^{2}$ flasks in complete medium (DermaLife ${ }^{\circledR}$ K Medium Kit, Lifeline Cell Technology,
Frederick, MD, USA) consisting of DMEM, 10\% fetal bovine serum (FBS), and $100 \mathrm{U} / \mathrm{mL}$ penicillin and streptomycin. Cells were trypsinized and passaged at $80-90 \%$ confluence. The cells were reseeded into 96 -well plates or 6-well plates at a density of $1 \times 10^{4}$ or $1 \times 10^{6}$ cells/well, respectively, and cultured in a humidified atmosphere of $5 \% \mathrm{CO}_{2}$ and $95 \%$ air, at $37^{\circ} \mathrm{C}$.

\section{Cell viability assay}

BM-MSCs were plated into 96-well plates at a density of $1 \times 10^{4}$ per well. After 2 days, cells were stimulated with icariin at concentrations of $0,0.001$, $0.01,0.1,1$ or $10 \mu \mathrm{M}$ for $24 \mathrm{~h}$. Cell proliferation was investigated using the 3-(4, 5-dimethylthiazolyl-2)-2, 5-diphenyltetrazolium bromide (MTT) assay. To perform the assay, $20 \mu \mathrm{L}$ of MTT was added to every well, and the plates were placed into a $5 \% \mathrm{CO}_{2}$-humidified incubator at $37^{\circ} \mathrm{C}$ for $4 \mathrm{~h}$. After incubation, supernatants were removed from the plates and $100 \mu \mathrm{L}$ of DMSO solution was added for $15 \mathrm{~min}$. The plates were read on a micro-plate reader at a wavelength of $492 \mathrm{~nm}$.

\section{RNA preparation and RT-PCR analysis}

BM-MSCs were seeded into 6-well plates at a density of $1 \times 10^{6}$ cells per well. Total RNA was extracted from the cells using TRIzol ${ }^{\mathbb{R}}$ reagent according to the manufacturer's instructions (Life Technologies, Carlsbad, CA, USA) after stimulation. First strand cDNA was synthesized using a Reverse Transcription System Kit (Tiangen Biotech, Beijing, China). RNA was reverse transcribed into cDNA and analyzed by RT-PCR using an ABI PRISM ${ }^{\circledR} 7000$ sequence detector (Applied Biosystems, Foster City, CA, USA). CREB, PKA, BMP2, Smad1, and Runx 2 mRNA levels were detected by real-time PCR in a reaction mixture of $20 \mu \mathrm{L}$ total volume containing $10 \mu \mathrm{L}$ of $2 \times$ SYBR $^{\circledR}$ TransStar Green PCR Super Mix, $0.6 \mu$ L sense and antisense primers, and $50 \mathrm{ng}$ RT product. The utilized primer sequences are shown in Table I.

TABLE I - Primer for real-time PCR assays

\begin{tabular}{lcc}
\hline Genes & \multicolumn{1}{c}{ Forward primer } & Reverse primer \\
\hline CREB & 5'-CTGAGGAGCTTGTACCACCG-3' & 5'-TGGATACCTGGGCTAATGTGG-3' \\
PKA & 5'-GAGCAGGAGAGCGTGAAAGAG-3' & 5'-GGGCTGTATTCTGAGAAGGGG-3' \\
BMP2 & 5'-GGGACCCGCTGTCTTCTAGT & 5'-TCAACTCAAATTCGCTGAGGAC-3' \\
Smad1 & 5'-ACCTGTGGCTTCCGTCTC-3' & 5'-ATCGTGGCTCCTTCGTC-3' \\
RUNX2 & 5'-GGGAACCAAGAAGGCACAGA-3' & 5'-GGTGGAATGGATGGATGGGG-3' \\
$\beta$-actin & 5'-CGCGAGTACAACCTTCTTGC-3' & 5'-CGTCATCCATGGCGAACTGG-3' \\
\hline
\end{tabular}




\section{Western blot analysis}

Cells were lysed with lysis buffer $(20 \mathrm{mM}$ Tris$\mathrm{HCl}, \mathrm{pH} 8.0,120 \mathrm{mM} \mathrm{NaCl}, 1 \%$ [v/v] Triton ${ }^{\mathrm{TM}} \mathrm{X}-100$, $10 \mathrm{mM}$ ethylenediaminetetraacetic acid, $1 \mathrm{mM}$ ethylene glycol tetraacetic acid, $0.05 \%$ [v/v] 2-mercaptoethanol, and $1 \times$ protease inhibitor solution). Cell debris was removed by centrifugation at $14,000 \times g$ for $15 \mathrm{~min}$, and equal amounts of protein extracted from each sample were heat denatured and subjected to sodium dodecyl sulfate polyacrylamide gel electrophoresis (SDS-PAGE) on a 10\% (v/v) polyacrylamide gel. After electrophoresis, proteins were electrotransferred onto polyvinylidene difluoride membranes. The membranes were blocked with $5 \%(\mathrm{w} / \mathrm{v})$ skimmed milk at room temperature for $2 \mathrm{~h}$ and probed with primary antibody at $4{ }^{\circ} \mathrm{C}$ overnight. The primary antibodies used were CREB, PKA, BMP2, Smad1, and RUNX2. After washing, membranes were incubated with the appropriate secondary antibody in 5\% (w/v) skimmed milk/Tris-buffered saline containing $0.05 \%$ Tween ${ }^{\circledR} 20$ for $1 \mathrm{~h}$ at $4^{\circ} \mathrm{C}$. Proteins were detected using SignalFire ${ }^{\mathrm{TM}}$ ECL Reagent (Cell Signaling Technology, Danvers, MA, USA).

\section{Enzyme-linked immunosorbent assay (ELISA) analysis}

Supernatants from BM-MSC cell culture were harvested at $24 \mathrm{~h}$ after stimulation, and centrifuged at $1000 \times g$ for $20 \mathrm{~min}$. The supernatants were analyzed for ALP by ELISA following the manufacturers' instructions (Nanjing Jiancheng Bioengineering Institute).

\section{Statistical analysis}

Data are expressed as the mean \pm SD of three independent determinations, and significance was analyzed using the $t$-test and one-way ANOVA. ${ }^{*} P<0.05$ was considered statistically significant. All analyses were performed using the Statistical Package for the Social Sciences (SPSS) statistical software for Windows, Version 18.0 (SPSS Inc., Chicago, IL, USA).

\section{RESULTS}

\section{Effect of icariin on cell viability}

The effect of icariin on cell viability of BM-MSCs was determined by MTT assay. As shown in Figure $1,0.001 \mu \mathrm{M}$ icariin had no significant effect on the proliferation of BM-MSCs. However, icariin at 0.01, 0.1, or $1 \mu \mathrm{M}$ promoted MSC proliferation $(P<0.01)$, and showed a quantitative relationship. Meanwhile, $10 \mu \mathrm{M}$ of icariin showed a certain cytotoxicity, significantly inhibiting the proliferation of BM-MSCs $(P<0.05)$.

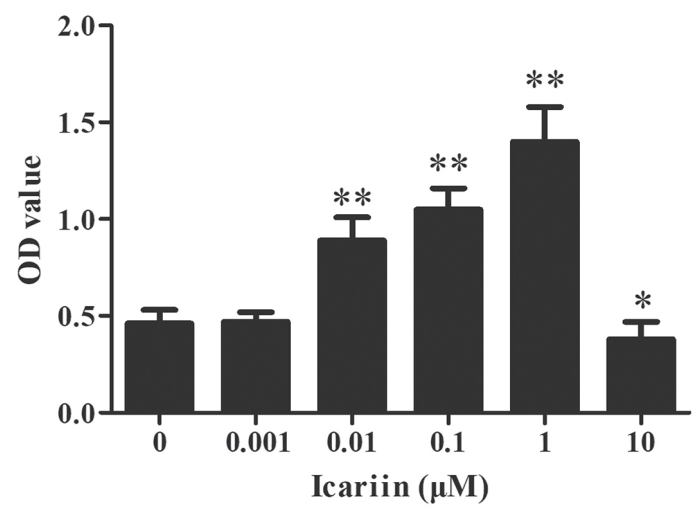

FIGURE 1 - Effects of different concentrations of icariin on the proliferation of BM-MSCs at 24 h. $v s$. treatment with $0 \mu \mathrm{M}$, ${ }^{*} P<0.05,{ }^{* *} P<0.01$.

\section{Assessment of ALP activity under different treatment conditions}

BM-MSCs were treated with different concentrations of icariin for $24 \mathrm{~h}$. the groups treated with $0.01,0.1$, or 1 $\mu \mathrm{M}$ icariin showed a significant increase in ALP activity compared with the control group $(P<0.05$, Figure $2 \mathrm{~A})$.

\section{Icariin administration enhanced BM-MSC gene and protein expression via the CAMP/PKA/CREB signaling pathway}

The cAMP/PKA/CREB signaling pathway has been shown to play an important role in differentiation of BM-MSCs. To determine the mechanism of icariin on BM-MSC differentiation, we examined the effect of icariin on the expression of components of the cAMP/PKA/ CREB signaling pathway. Our qPCR data showed that icariin significantly enhanced mRNA expression of $C R E B$ and $P K A$ in BM-MSCs. As shown in Figure 2, treatment with icariin at a concentration of $1 \mu \mathrm{M}$ induced the most significant effect with a 1.9-fold increase in expression of $C R E B$ and a 1.6-fold increase in $P K A$, compared to the blank group $(0 \mu \mathrm{M})$. The effect of icariin on BM-MSC mRNA expression showed a dose-response relationship (Figure 2. C, D). Finally we examined the effect of icariin on protein expression of PKA/CREB signaling molecules, and found that icariin stimulation of BM-MSCs showed a dose-response relationship. Expression of PKA/CREB was significant increased after treatment with icariin at the concentration of $1 \mu \mathrm{M}$, including both mRNA and protein levels (Figure $2 \mathrm{~B}$ ). Taken together, these 
results demonstrate that icariin may regulate BM-MSC differentiation through activation of the cAMP/PKA/ CREB signaling pathway.

\section{Icariin induced BMP2, Smad1, and RUNX2 mRNA and protein expression in BM-MSCs}

The CREB receptors BMP2, Smad1, and Runx2 were constitutively expressed on BM-MSCs (Figure 3). The mRNA and protein levels of BMP2, Smad1, and Runx 2 were analyzed after treatment of BM-MSCs with icariin to determine whether icariin increased BMP2, Smad1, and Runx2 expression. Analysis by RT-PCR showed that BM-MSCs treated with icariin exhibited a significant and dose-dependent increase in the mRNA expression of BMP2, Smad1, and Runx2, compared with untreated cells (Figure $3 \mathrm{~A}-\mathrm{C}$ ). The effect of icariin on BMP2, Smad1, and Runx2 protein expression was also dose-dependent. Meanwhile, the mRNA and protein expression of BMP2, Smad1, and RUNX2 showed a significantly greater sensitivity to icariin $(1 \mu \mathrm{M})$ stimulation than untreated cells (all $P<0.01$ ).
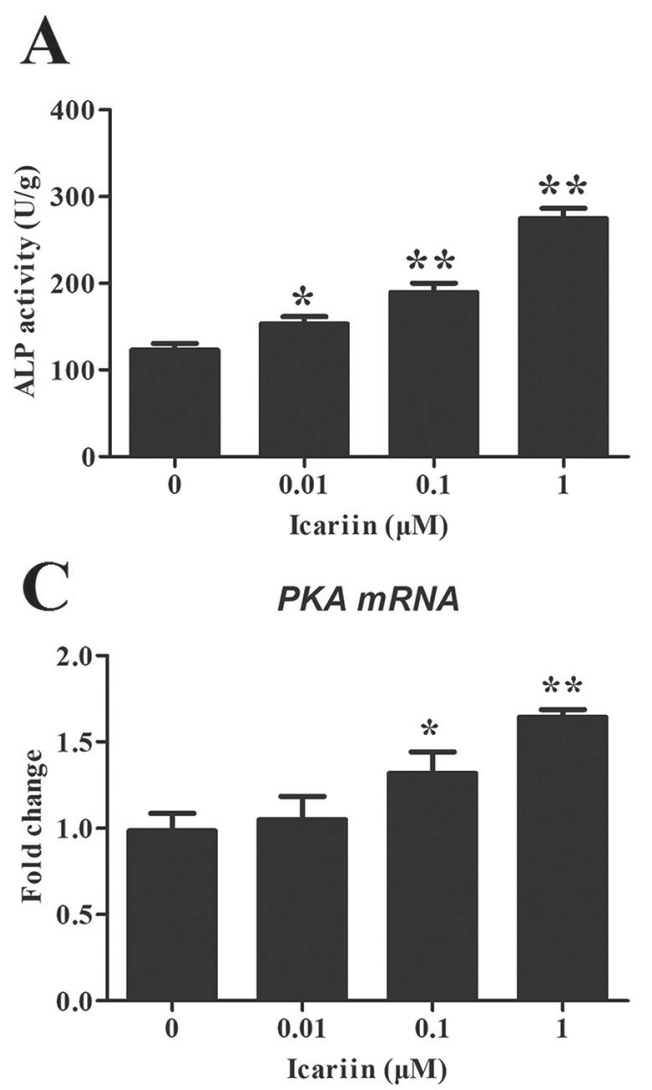

\section{DISCUSSION}

The herb Epimedium has long been used in traditional Chinese medicine to treat bone fractures and prevent osteoporosis. Researchers believe that the flavonoids contained in the herb are the effective component responsible for this activity. Icariin should be an effective agent for bone-strengthening activity, and one of its mechanisms is to stimulate the proliferation and enhance the osteogenic differentiation of MSCs (Chen et al., 2005). Icariin has been shown to increase osteogenic differentiation and mineralization of BMSCs and osteoblasts, and to inhibit osteoclast formation and bone resorption activity (Zhang et al., 2011; Ma et al., 2014; Zhang et al., 2016). In this paper, we found that low concentrations $(0.01,0.1,1 \mu \mathrm{M})$ of icariin stimulated BM-MSC proliferation $(P<0.01)$, but that $10 \mu \mathrm{M}$ icariin had a certain cytotoxicity, and significantly inhibited the proliferation of BM-MSCs $(P<0.05)$.

Hormones that stimulate the synthesis of cAMP regulate many cell type-specific processes including gene transcription, cell proliferation, differentiation,
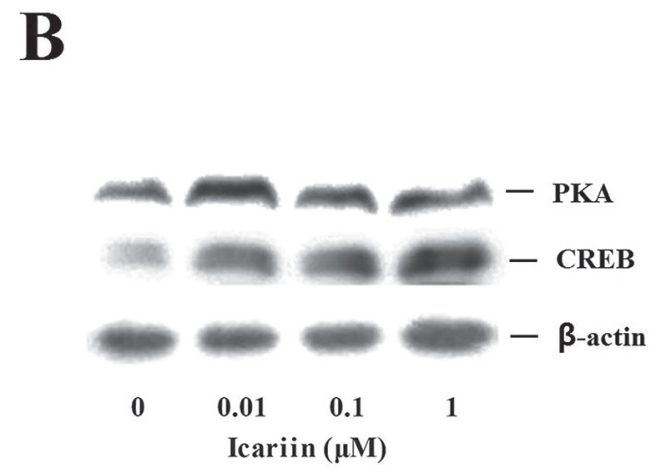

D CREB $m R N A$

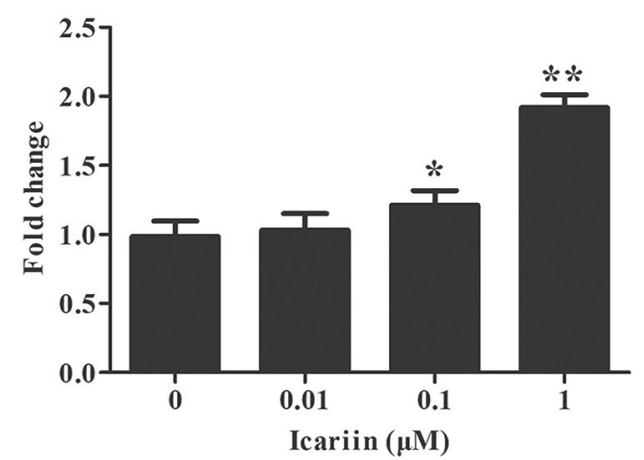

FIGURE 2 - Icariin actives the cAMP/PKA/CREB signaling pathway in BM-MSCs. (A) Icariin showed a dose-dependent effect on alkaline phosphatase (ALP) activity in BM-MSCs. (B) WB analysis showed that icariin treatment of BM-MSCs significantly enhanced protein expression of PKA and CREB. (C)(D) RT-PCR results showed that icariin dose-dependently increased the mRNA levels of $P K A$ and CREB in BM-MSCs. vs. treatment with $0 \mu \mathrm{M},{ }^{*} P<0.05,{ }^{* *} P<0.01$. 


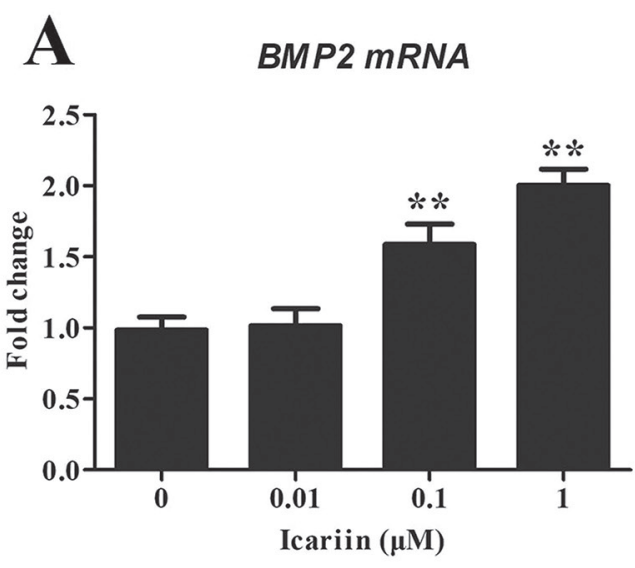

Runx2 mRNA

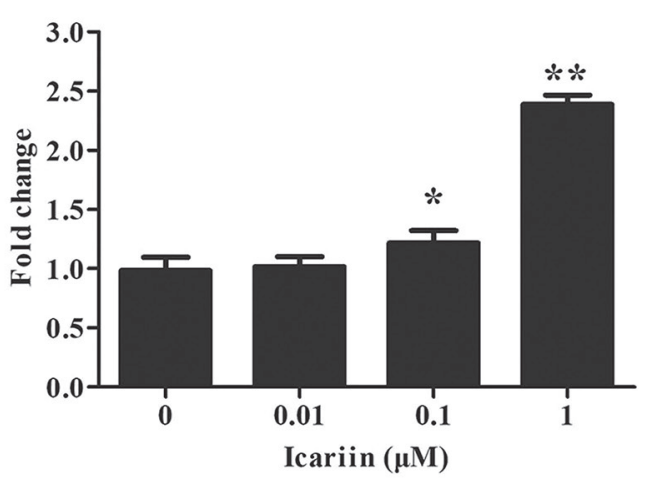

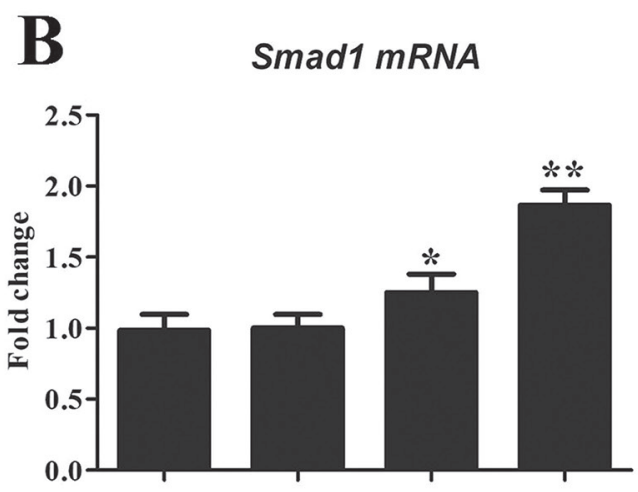

D

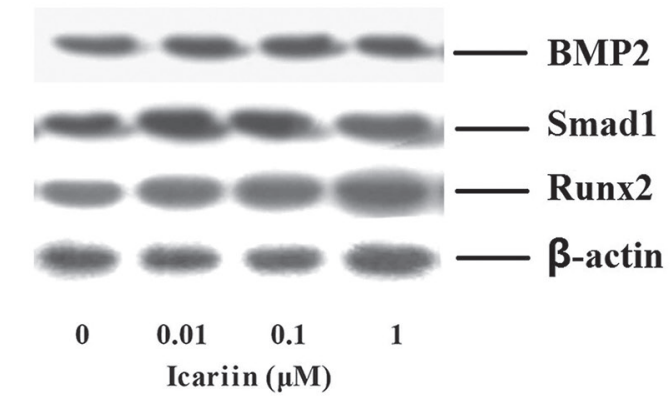

FIGURE 3 - Icariin induced BM-MSC differentiation by BMP2, Smad1, and Runx2. RT-PCR and WB results indicated that icariin significantly increased the expression of BMP2, Smad1, and Runx2 in BM-MSCs. vs. treatment with $0 \mu \mathrm{M} ;{ }^{*} P<0.05,{ }^{* *} P<0.01$

and survival. These processes are mediated, in part, by the MAP (mitogen-activated protein) kinase (or ERK; extracellular signal response kinase) cascade in a wide range of diverse cell types including pancreatic islet cells, bone cells, pituitary cells, thyroid cells, neuronal cells and others (Li et al., 2016). In this study, we found that cAMP consistently enhanced the expression of the osteogenesis marker ALP. The results of cell proliferation assay demonstrated that icariin stimulated BM-MSC proliferation. These results indicated that icariin treatment at different concentrations promoted proliferation of BM-MSCs through the cAMP/PKA/ CREB pathway. Further, we sought to examine the effect of icariin on expression of PKA/CREB mRNA and protein, by analyzing the mRNA and protein levels of PKA/CREB after treatment of BM-MSCs. Analysis using RT-PCR and WB showed that BM-MSCs treated with icariin exhibited a dose-dependent increase in the mRNA and protein expression of PKA/CREB, compared with untreated cells. Several previous studies have evaluated the role of the PKA pathway in osteogenic differentiation of hMSCs, and reported that pretreatment of human MSCs with a cAMP analog or forskolin enhanced bone formation. cAMP is a pivotal intracellular signaling molecule, the main function of which is to activate cAMP-dependent PKA (Yang et al., 2008). Adenylate cyclase is activated through dissociation of G-proteins, causing the conversion of adenosine triphosphate into cAMP. Subsequently, cAMP activates PKA, which in turn phosphorylates CREB protein, and this translocates into the nucleus where it activates transcription of target genes ( Kim et al., 2013; Chen et al., 2016). However, precisely how the administration of icariin regulates osteogenic differentiation of BM-MSCs by the $\mathrm{CAMP} / \mathrm{PKA} / \mathrm{CREB}$ signaling pathway requires further elucidation. In the current study, cell proliferation assays, osteogenic gene expression testing, and ALP activity detection were used in order to determine the underlying molecular mechanism by which icariin administration modulates the cAMP/ PKA/CREB signaling pathway.

Previous studies have shown that activation of the PKA pathway synergistically participates in BMP-2induced osteoblastic differentiation, possibly by mediating the CREB and/or Ras/MAPK pathways, and activation of the PKA pathway may be one of the key BMP-2-activated signaling events that lead to osteogenic differentiation 
(Zhao et al., 2006). Based on these findings, we also tested the mRNA and protein expression of BMP2, Smad1, and Runx2 in BM-MSCs stimulated with icariin to investigate whether the administration of icariin regulates osteogenic differentiation of BM-MSCs via the cAMP/PKA/CREB signaling pathway. BMP/Smad signals are mediated by BMP receptors (type I and II). Smad1, 5, and 8 are phosphorylated by the BMP receptors and form a complex with Smad4, following which the complex is translocated into the nucleus. Within the nucleus, the phosphorylated Smads interact with other transcription factors such as Runx2 to initiate transcription of osteogenic-related genes (Xu et al., 2015). We focused on signaling pathways involved in icariin-mediated BMP2, Smad1, and Runx2 expression in BM-MSCs according to previous reports (Guo et al., 2016; Su et al., 2015). Analysis by RT-PCR and WB showed that BMP2, Smad1, and Runx2 were activated by icariin in BM-MSCs (Figure 3). Taken together, our results indicate that icariin regulates the expression of BMP2 in BM-MSCs through the cAMP/ PKA/CREB signaling pathway.

\section{ACKNOWLEDGMENTS}

The authors are grateful to the National Natural Science Foundation of China (31370764) for their support of this work.

\section{REFERENCES}

Bianco P, Riminucci M, Gronthos S, Robery PG. Bone marrow stromal stem cells: nature, biology, and potential applications. Stem Cells. 2001;19(2):180-92.

Carrington JL. Aging bone and cartilage: cross-cutting issues. Biochem Biophys Res Commun. 2005;328(3):700-8.

Chen B, Lin T, Yang X, Li Y, Xie D, Cui H. Intermittent parathyroid hormone (1-34) application regulates cAMPresponse element binding protein activity to promote the proliferation and osteogenic differentiation of bone mesenchymal stromal cells, via the cAMP/PKA signaling pathway. Exp Ther Med. 2016;11(6):2399-406.

Chen KM, Ge BF, Ma HP, Liu XY, Bai MH, Wang Y. Icariin, a flavonoid from the herb Epimedium enhances the osteogenic differentiation of rat primary bone marrow stromal cells. Pharmazie. 2005;60(12):939-42.
Dazzi F, Ramasamy R, Glennie S, Jones SP, Roberts I. The role of mesenchymal stem cells in haemopoiesis. Blood Rev. 2006;20(3):161-71.

Guo C, Li C, Yang K, Kang H, Xu X, Xu X, et al. Increased EZH2 and decreased osteoblastogenesis during local irradiationinduced bone loss in rats. Sci Rep. 2016;6:31318.

Hsieh TP, Sheu SY, Sun JS, Chen MH, Liu MH. Icariin isolated from Epimedium pubescens regulates osteoblasts anabolism through BMP-2, SMAD4, and Cbfa1 expression. Phytomedicine. 2010;17(6):414-23.

Huang JM, Bao Y, Xiang W, Jing XZ, Guo JC, Yao XD, et al. Icariin regulates the bidirectional differentiation of bone marrow mesenchymal stem cells through canonical Wnt signaling pathway. Evid-Based Compl Alt Med. 2017;2017:8085325.

Ivanova S, Vasileva L, Ivanova S, Peikova L, Obreshkova D. Osteoporosis: therapeutic options. Folia Med. 2015;57(34):181-90.

Kim JM, Choi JS, Kim YH, Jin SH, Lim S, Jang HJ, et al. An activator of the cAMP/PKA/CREB pathway promotes osteogenesis from human mesenchymal stem cells. J Cell Physiol. 2013;228(3):617-26.

Li X, Xu Z, Bai J, Yang S, Zhao S, Zhang Y, et al. Umbilical cord tissue-derived mesenchymal stem cells induce t lymphocyte apoptosis and cell cycle arrest by expression of indoleamine 2, 3-Dioxygenase. Stem Cells Int. 2016;2016:7495135.

Li Y, Dillon TJ, Takahashi M, Earley KT, Stork PJ. Protein kinase A-independent ras activation cooperates with Rap1 to mediate activation of Extracellular signal-regulated kinases (ERK) by cAMP. J Bio Chem. 2016;291(41):21584-21595.

Luo Z, Liu M, Sun L, Rui F. Icariin recovers the osteogenic differentiation and bone formation of bone marrow stromal cells from a rat model of estrogen deficiency-induced osteoporosis. Mol Med Rep. 2015;12(1):382-8.

Ma HP, Ma XN, Ge BF, Zhen P, Zhou J, Gao YH, et al. Icariin attenuates hypoxia-induced oxidative stress and apoptosis in osteoblasts and preserves their osteogenic differentiation potential in vitro. Cell Proliferat. 2014;47(6):527-39.

Su X, Liao L, Shuai Y, Jing H, Liu S, Zhou H, et al. MiR-26a functions oppositely in osteogenic differentiation of BMSCs and ADSCs depending on distinct activation and roles of Wnt and BMP signaling pathway. Cell Death Dis. 2015;6:e1851. 
Wu Y, Cao L, Xia L, Wu Q, Wang J, Wang X, et al. Evaluation of osteogenesis and angiogenesis of icariin in local controlled release and systemic delivery for calvarial defect in ovariectomized rats. Sci Rep. 2017;7(1):5077.

Xu L, Liu Y, Hou Y, Wang K, Wong Y, Lin S, et al. U0126 promotes osteogenesis of rat bone-marrow-derived mesenchymal stem cells by activating BMP/Smad signaling pathway. Cell Tissue Res. 2015;359(2):537-45.

Yang DC, Tsay HJ, Lin SY, Chiou SH, Li MJ, Chang TJ, et al. cAMP/PKA regulates osteogenesis, adipogenesis and ratio of RANKL/OPG mRNA expression in mesenchymal stem cells by suppressing leptin. Plos One. 2008;3(2):e1450.

Zhang D, Fong C, Jia Z, Cui L, Yao X, Yang M. Icariin stimulates differentiation and suppresses adipocytic transdifferentiation of primary osteoblasts through estrogen receptor-mediated pathway. Calcified Tissue Int. 2016;99(2):187-98.
Zhang J, Li Y, Sun J, Liu C, Zhang D. Synergistic or antagonistic effect of MTE plus TF or icariin from Epimedium koreanum on the proliferation and differentiation of primary osteoblasts in vitro. Biol Trace Element Res. 2011;143(3):1746-57.

Zhao L, Li G, Zhou GQ. SOX9 directly binds CREB as a novel synergism with the PKA pathway in BMP-2-induced osteochondrogenic differentiation. J Bone Miner Res. 2009;24(5):826-36.

Zhao L, Yang S, Zhou GQ, Yang J, Ji D, Sabatakos G, et al. Downregulation of cAMP-dependent protein kinase inhibitor gamma is required for BMP-2-induced osteoblastic differentiation. Int J Biochem Cell B. 2006;38(12):2064-73.

Received for publication on $20^{\text {th }}$ April 2018 Accepted for publication on $21^{\text {st }}$ June 2018 